\title{
Vitamin D Levels in Subjects with Prostate Cancer Compared to Age-Matched Controls
}

\author{
Subhashini Yaturu, ${ }^{1}$ Sonya Zdunek, ${ }^{2}$ and Barbara Youngberg ${ }^{3}$ \\ ${ }^{1}$ Department of Endocrinology, Stratton VA Medical Center, Albany, NY 12208, USA \\ ${ }^{2}$ IT Department, Stratton VA Medical Center, Albany, NY 12208, USA \\ ${ }^{3}$ Department of Research, Stratton VA Medical Center, Albany, NY 12208, USA
}

Correspondence should be addressed to Subhashini Yaturu, subhashini.yaturu@va.gov

Received 19 October 2012; Revised 25 November 2012; Accepted 27 November 2012

Academic Editor: Manfred P. Wirth

Copyright (C) 2012 Subhashini Yaturu et al. This is an open access article distributed under the Creative Commons Attribution License, which permits unrestricted use, distribution, and reproduction in any medium, provided the original work is properly cited.

Prostate cancer $(\mathrm{PCa})$ is the second most common cancer in men worldwide and the second leading cause of cancer deaths in men in the United States. Vitamin D is considered to have anticancer properties, currently thought to work mainly through its nuclear receptor or vitamin D receptor. In this retrospective study, we compared vitamin D levels in subjects with PCa with those of age-matched men without PCa. Study subjects included 479 in each group with a mean age of 73 and a mean creatinine of 1.05 and 1.15. Levels of $25(\mathrm{OH})$ vitamin D were $28.4 \pm 0.54$ and $28.05 \pm 0.62$ in subjects with and without PCa. Levels of 1,25 $(\mathrm{OH})$ vitamin D were $47.2 \pm 6.8$ and $47.1 \pm 7.11$ in subjects with and without PCa. In contrast to other studies, we did not find a significant difference in vitamin D levels. Among prostate cancer patients, vitamin D levels correlated positively with age $(r=0.12$, $P<0.02)$, and were negatively associated with BMI $(r=-0.13, P=0.003)$, glucose $(r=-0.12, P<0.007), \mathrm{HbA1C}(r=-0.16$, $P=0.001)$, and PTH $(r=-0.21 ; P<0.0001)$. The data do not show the causal effect of vitamin D levels on PCa.

\section{Introduction}

Prostate cancer is the most commonly diagnosed cancer in men in the United States [1]. It is estimated that 241,740 men will be diagnosed with and 28,170 men will die of cancer of the prostate in 2012 [1]. Vitamin D from the skin and diet is metabolized in the liver to 25-hydroxyvitamin $\mathrm{D}(25(\mathrm{OH}) \mathrm{D})$, which is used to determine a patient's vitamin D status [2]. Vitamin D insufficiency affects almost $50 \%$ of the population worldwide $[2,3]$. In the Third National Health and Nutrition Examination Survey (NHANES III), a cross-sectional multivariate analysis, the lowest quartile of $25(\mathrm{OH}) \mathrm{D}$ level $(<17.8 \mathrm{ng} / \mathrm{mL})$ was independently associated with all-cause mortality in the general population [4]. Prostate cells contain vitamin $\mathrm{D}$ receptors as well as enzymes necessary for vitamin D metabolism. Vitamin D metabolites are considered to have an antiproliferative and a prodifferentiating effect on prostate cancer cell lines in vitro and in vivo. Low levels of plasma vitamin $\mathrm{D}$ have been implicated as a possible risk factor for both prostate cancer incidence and advanced disease.
In a study to examine the association between vitamin $\mathrm{D}$ receptor (VDR) polymorphisms and prostate cancer stage, the authors concluded that low levels of vitamin D may increase the risk of prostate cancer progression [2]. However, meta-analysis of published literature of studies on vitamin $D$ levels and association with prostate cancer reported no association $[5,6]$ or little evidence to support a major role of vitamin $\mathrm{D}$ in preventing prostate cancer or its progression [7]. There are on-going clinical trials to evaluate the benefit of vitamin $\mathrm{D}$ in prostate cancer prevention and progression as noted at clinical trials.gov. No studies have compared the vitamin D levels to age-matched controls. The Institute of Medicine's 2011 report on dietary reference intakes for calcium and vitamin D could not make any conclusion regarding whether vitamin $\mathrm{D}$ has anticancer activities in humans [4]. Since prostate cancer is common in the elderly and vitamin $\mathrm{D}$ deficiency is common in that age group, the association of vitamin D deficiency with prostate cancer may be a simple association with age, not necessarily a factor in the cause or progression of prostate cancer. The aim of this 
study is to evaluate vitamin D levels in subjects with prostate cancer compared to age-matched controls.

\section{Materials and Methods}

This is a clinical, retrospective study to evaluate vitamin $\mathrm{D}$ levels in subjects with or without prostate cancer. This study was approved by the Institutional Review Board as well as Research and Development at Stratton VA Medical Center at Albany, NY, USA. Subjects included patients whose vitamin D levels had been analyzed at Veterans Integrated Service Network (VISN-2) of Veterans Health Administration (VHA) between 2006 and 2009. Patients with endstage kidney disease and chronic kidney disease, defined as having serum creatinine levels greater than $2.5 \mathrm{mg} / \mathrm{dL}$, were excluded. Using the Current Procedural Terminology (CPT) codes for diagnosis of prostate cancer and the exclusion criteria for the study, 479 subjects with a diagnosis of prostate cancer were identified from review of approximately 5,000 patient records. The dates of diagnosis of prostate cancer were from 1994 to 2011. An additional 479 subjects without diagnoses of prostate cancer were age matched to the prostate cancer group and included in the study.

The following data was retrieved from the Computerized Patient Record System for all subjects: age, BMI, history of diabetes and hypertension, HbA1C, glucose, creatinine, estimated glomerular filtration rate (eGFR), $25(\mathrm{OH})$ vitamin $\mathrm{D}$; 1,25 $(\mathrm{OH})$ vitamin D levels, calcium, and PTH.

2.1. Statistical Analysis. The data is presented as \pm SD. A $P$ value of $<0.05$ is considered significant. The difference in vitamin $\mathrm{D}$ levels in subjects with prostate cancer compared to age-matched control subjects without prostate cancer was evaluated by the Student's $t$-test (two-sided, heteroscedastic). Correlation of vitamin D levels with other clinical and biochemical parameters was calculated.

\section{Results}

Study subjects include 479 with prostate cancer and 473 subjects without prostate cancer. Clinical data of subjects with and without prostate cancer is shown in Table 1 . The groups are age matched. There is no significant difference in BMI, or in the percent of subjects with hypertension. Biochemical data of subjects with and without prostate cancer is shown in Table 2. There is no significant difference in the vitamin D levels (both 25(OH) vitamin D and 1,25 $(\mathrm{OH})$ vitamin D levels) between the subjects with prostate cancer compared to the age-matched subjects without prostate cancer $(t=$ $0.6808 ; P=0.000595)$. In the combined analysis of vitamin $\mathrm{D}$ in all subjects (with and without prostate cancer), we observed significant negative correlations between vitamin D levels and BMI $(r=-0.11 ; P<0.0005)$ and PTH levels $(r=-0.26 ; P<0.0001)$. In the subgroup analysis of the data of subjects with prostate cancer, we found statistically significant correlations of vitamin $\mathrm{D}$ with age $(r=0.12 ; P<$ $0.02)$, BMI $(r=-0.13 ; P=0.003)$, diagnosis of diabetes $(r=$ $-0.14 ; P<0.01)$, HbA1C $(r=-0.16 ; P=0.0008$, glucose $(r=-0.12 ; P<0.007)$, and PTH $(r=-0.21 ; P<0.0001)$.
TABLE 1: Clinical parameters in subjects with prostate cancer compared to age-matched controls without prostate cancer.

\begin{tabular}{lccc}
\hline \multicolumn{4}{c}{ Clinical data in subjects with and without prostate cancer } \\
& P. Ca & No P. Ca & $P$ value \\
\hline Age (years) & $73 \pm 0.4$ & $74 \pm 0.4$ & 0.81 \\
BMI & $29.1 \pm 0.23$ & $29.3 \pm 0.27$ & 0.66 \\
DM (\%) & 36 & 42 & 0.05 \\
HTN (\%) & 85 & 86 & 0.77 \\
\hline
\end{tabular}

P. Ca: prostate cancer; BMI: body mass index; DM: diabetes; HTN: Hypertension.

TABle 2: Biochemical data in subjects with prostate cancer compared to age matched controls without Prostate cancer.

\begin{tabular}{lccc}
\hline \multicolumn{4}{c}{ Biochemical data in subjects with and without prostate cancer } \\
& P. Ca & No P. Ca & P value \\
\hline Vitamin D (ng/dL) & $28.4 \pm 0.53$ & $28.1 \pm 0.63$ & 0.68 \\
Creatinine & $1.15 \pm 0.02$ & $1.07 \pm 0.02$ & 0.02 \\
eGFR (mL/min) & $72 \pm 0.86$ & $81 \pm 1.3$ & $<0.001$ \\
Calcium (mg/dL) & $9.5 \pm 0.01$ & $9.5 \pm 0.02$ & NS \\
Glucose (mg/dL) & $114 \pm 1.6$ & $119 \pm 2$ & 0.03 \\
HbA1C & $6.4 \pm 0.057$ & $6.6 \pm 0.07$ & 0.07 \\
PTH (pg/mL) & $74 \pm 7$ & $81 \pm 7$ & 0.53 \\
$1,25,(\mathrm{OH})$ Vit. D (pg/mL) & $47 \pm 6.8$ & $47 \pm 7.1$ & 0.99 \\
\hline
\end{tabular}

P. Ca: prostate cancer; eGFR: calculated glomerular filtration rate; PTH: Parathyroid hormone; $1,25,(\mathrm{OH})$ Vit. D: $1,25(\mathrm{OH})$ vitamin $\mathrm{D} ; P<0.05$ is considered significant.

\section{Discussion}

The most important point in the study is that there is no significant difference in the vitamin D levels between the subjects with and without prostate cancer when age matched. These results vary from epidemiological observations that have suggested links between low vitamin D and increased risk of prostate cancer. Calcitriol, the biologically active form of vitamin $\mathrm{D}(1,25(\mathrm{OH}) \mathrm{D})$, has been shown to be an antiproliferative, prodifferentiation, proapoptotic agent, and an inhibitor of cell migration. Vitamin D deficiency is associated with an increased risk and poor prognosis of several types of cancer. Meta-analysis of genomic studies on the association between the two most studied VDR polymorphisms (FokI and BsmI) have shown significantly higher risk of cancer when the researchers pooled estimates from cancer sites possibly associated with vitamin D levels (prostate, breast, skin, ovary, non-Hodgkin lymphoma) [8]. In the Third National Health and Nutrition Examination Survey (NHANES III, USA), serum $25(\mathrm{OH}) \mathrm{D}$ levels less than $17.8 \mathrm{ng} / \mathrm{mL}$ were associated with a $26 \%$ increased rate of all-cause mortality (mortality rate ratio $(95 \% \mathrm{CI}:+8 \% ;+46 \%)$ ) [4].

Our results, showing no correlation between vitamin D levels and prostate cancer, are similar to recent data of some publications. The findings of the NIH epidemiological study noted that this large prospective study did not support the hypothesis that vitamin $\mathrm{D}$ is associated with decreased risk of prostate cancer; indeed, higher circulating $25(\mathrm{OH}) \mathrm{D}$ concentrations may be associated with increased risk of 
aggressive disease [9]. In a study from California, Tseng and associates did not find any association of $1,25(\mathrm{OH})_{2} \mathrm{D}$ to prostate cancer [10]. In a case control study from Sweden, there was no association between prostate cancer risk and vitamin D levels [11].

Ultraviolet-induced synthesis of vitamin $\mathrm{D}_{3}$ in human skin is dependent on several factors, such as time spent out-of-doors, skin pigmentation, and age of subjects, as endogenous skin synthesis capacity decreases with age and sun protection habits, mainly in populations where clothing (garments and veils) covers most of the skin surface. Dietary intake of vitamin D increases serum levels of 25-hydroxyvitamin D [12]. 25(OH)D constitutes the bulk of vitamin $\mathrm{D}$ reserves. With supraphysiologic dose administration, large quantities of vitamin $\mathrm{D}(3)$ are stored as the native compound, presumably in body fat, and are slowly released to be converted to $25(\mathrm{OH}) \mathrm{D}$ [13]. Because of its relatively long half-life $\left(\tau_{1 / 2}=12.9(\mathrm{SD}: 3.6 \mathrm{~d})\right)$, the serum $25(\mathrm{OH}) \mathrm{D}$ level is considered as the best gauge of individual vitamin $\mathrm{D}$ status [14]. Elderly people are at high risk for vitamin D deficiency if their life style entails few outdoor activities, their skin is thick, and they exhibit impairment of renal function [15].

Our data on all subjects combined is consistent with the results of several publications that the vitamin $\mathrm{D}$ levels negatively correlate with BMI $[16,17]$. We noted that a lower number of subjects with prostate cancer had diabetes in our study. This is consistent with the epidemiological evidence to show that subjects with diabetes have lower rates of prostate cancer [18].

In the subanalysis of the data from prostate cancer subjects, we noted association of vitamin D levels with glucose and HbA1C levels. Most of the subjects with prostate cancer receive androgen deprivation therapy. Metabolic effects of androgen deprivation therapy for prostate cancer include insulin resistance, diabetes, dyslipidemia, adverse body composition, and metabolic syndrome that contribute to increase in cardiovascular mortality in this population $[19,20]$. Kim and associates reported that men with higher HbAlc levels presented with more biologically aggressive prostate tumors at radical prostatectomy [21]. Similar evidence was noted by Hong and associates that although simple history of having DM may not be a significant factor regarding aggressiveness of clinically localized prostate cancer, the glycemic control, as represented by $\mathrm{HbA} 1 \mathrm{C}$ level, may be a useful preoperative predictor of aggressive tumor profile among patients with DM who are also diagnosed with clinically localized prostate cancer [22].

Loss of bone mineral density is a well-known consequence of androgen deprivation therapy in men with prostate cancer $[23,24]$. Review of the results of 12 clinical trials was said to have shown that, at the doses commonly recommended, 500-1,000 $\mathrm{mg}$ calcium and 200-500 IU vitamin $\mathrm{D}$ per day, men undergoing androgen deprivation lose bone mineral density [23].

4.1. Drawbacks. This was a retrospective study. Comorbidities were not looked into. In some cases, vitamin D analysis was done prior to the diagnosis of prostate cancer and in others, after the diagnosis of prostate cancer. There was no attempt to account for this or compare results based on when the vitamin D levels were measured.

\section{Conclusions}

Vitamin D levels are similar in elderly men with or without prostate cancer.

\section{Disclaimer}

The views expressed in this paper are those of the authors and do not necessarily reflect the position or policy of the Department of Veterans Affairs or the United States government.

\section{Acknowledgments}

This material is the result of work supported with resources and the use of facilities at the Stratton VA Medical Center at Albany, New York. S. Yaturu receives salary support from VA.

\section{References}

[1] http://www.cdc.gov/cancer/prostate/index.htm.

[2] M. F. Holick, "Medical progress: vitamin D deficiency," New England Journal of Medicine, vol. 357, no. 3, pp. 266-281, 2007.

[3] R. Nair and A. Maseeh, "Vitamin D: the "sunshine" vitamin," Journal of Pharmacology and Pharmacotherapeutics, vol. 3, no. 2, pp. 118-126, 2012.

[4] M. L. Melamed, E. D. Michos, W. Post, and B. Astor, "25hydroxyvitamin D levels and the risk of mortality in the general population," Archives of Internal Medicine, vol. 168, no. 15, pp. 1629-1637, 2008.

[5] S. Gandini, M. Boniol, J. Haukka et al., "Meta-analysis of observational studies of serum 25-hydroxyvitamin D levels and colorectal, breast and prostate cancer and colorectal adenoma," International Journal of Cancer, vol. 128, no. 6, pp. 1414-1424, 2011.

[6] L. Yin, E. Raum, U. Haug, V. Arndt, and H. Brenner, "Metaanalysis of longitudinal studies: serum vitamin D and prostate cancer risk," Cancer Epidemiology, vol. 33, no. 6, pp. 435-445, 2009.

[7] R. Gilbert, R. M. Martin, R. Beynon et al., "Associations of circulating and dietary vitamin $\mathrm{D}$ with prostate cancer risk: a systematic review and dose-response meta-analysis," Cancer Causes and Control, vol. 22, no. 3, pp. 319-340, 2011.

[8] S. Raimondi, H. Johansson, P. Maisonneuve, and S. Gandini, "Review and meta-analysis on vitamin D receptor polymorphisms and cancer risk," Carcinogenesis, vol. 30, no. 7, pp. 1170-1180, 2009.

[9] J. Ahn, U. Peters, D. Albanes et al., "Serum vitamin D concentration and prostate cancer risk: a nested case-control study," Journal of the National Cancer Institute, vol. 100, no. 11, pp. 796-804, 2008.

[10] M. Tseng, V. Giri, D. Watkins-Bruner, and E. Giovannucci, "Dairy intake and 1,25-dihydroxyvitamin D levels in men at high risk for prostate cancer," Cancer Causes and Control, vol. 20, no. 10, pp. 1947-1954, 2009.

[11] J. Brändstedt, M. Almquist, J. Manjer, and J. Malm, "Vitamin D, PTH, and calcium and the risk of prostate cancer: 
a prospective nested case-control study," Cancer Causes and Control, vol. 23, no. 8, pp. 1377-1385, 2012.

[12] A. Cranney, T. Horsley, S. O'Donnell et al., "Effectiveness and safety of vitamin D in relation to bone health," Evidence Report/Technology Assessment, no. 158, pp. 1-235, 2007.

[13] R. P. Heaney, L. A. G. Armas, J. R. Shary, N. H. Bell, N. Binkley, and B. W. Hollis, "25-Hydroxylation of vitamin $\mathrm{D}_{3}$ : relation to circulating vitamin $\mathrm{D}_{3}$ under various input conditions," American Journal of Clinical Nutrition, vol. 87, no. 6, pp. 17381742, 2008.

[14] M. W. J. Davie, D. E. M. Lawson, and C. Emberson, "Vitamin D from skin: contribution to vitamin D status compared with oral vitamin $\mathrm{D}$ in normal and anticonvulsant-treated subjects," Clinical Science, vol. 63, no. 5, pp. 461-472, 1982.

[15] A. Timpini, L. Pini, C. Tantucci, S. Cossi, and V. Grassi, "Vitamin D and health status in elderly," Internal and Emergency Medicine, vol. 6, no. 1, pp. 11-21, 2011.

[16] A. Jungert, H. J. Roth, and M. Neuhauser-Berthold, "Serum 25-hydroxyvitamin $\mathrm{D}_{3}$ and body composition in an elderly cohort from Germany: a cross-sectional study," Nutrition \& Metabolism, vol. 9, article 42, 2012.

[17] C. Halthur, A. L. V. Johansson, M. Almquist et al., "Serum calcium and the risk of prostate cancer," Cancer Causes and Control, vol. 20, no. 7, pp. 1205-1214, 2009.

[18] E. Giovannucci, D. M. Harlan, M. C. Archer et al., "Diabetes and cancer: a consensus report," Diabetes Care, vol. 33, no. 7, pp. 1674-1685, 2010.

[19] B. R. Shastri and S. Yaturu, "Metabolic complications and increased cardiovascular risks as a result of androgen deprivation therapy in men with prostate cancer," Prostate Cancer, vol. 2011, Article ID 391576, 9 pages, 2011.

[20] I. H. Derweesh, C. J. DiBlasio, M. C. Kincade et al., "Risk of new-onset diabetes mellitus and worsening glycaemic variables for established diabetes in men undergoing androgendeprivation therapy for prostate cancer," BJU International, vol. 100, no. 5, pp. 1060-1065, 2007.

[21] H. S. Kim, J. C. Presti, W. J. Aronson et al., "Glycemic control and prostate cancer progression: results from the SEARCH database," Prostate, vol. 70, no. 14, pp. 1540-1546, 2010.

[22] S. K. Hong, S. T. Lee, S. S. Kim et al., "Significance of preoperative HbA1c level in patients with diabetes mellitus and clinically localized prostate cancer," Prostate, vol. 69, no. 8, pp. 820-826, 2009.

[23] M. Datta and G. G. Schwartz, "Calcium and vitamin d supplementation during androgen deprivation therapy for prostate cancer: a critical review," Oncologist, vol. 17, no. 9, pp. 1171-1179, 2012.

[24] S. Yaturu, S. DjeDjos, G. Alferos, and C. Deprisco, "Bone mineral density changes on androgen deprivation therapy for prostate cancer and response to antiresorptive therapy," Prostate Cancer and Prostatic Diseases, vol. 9, no. 1, pp. 35-38, 2006. 


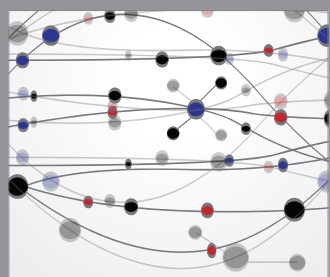

The Scientific World Journal
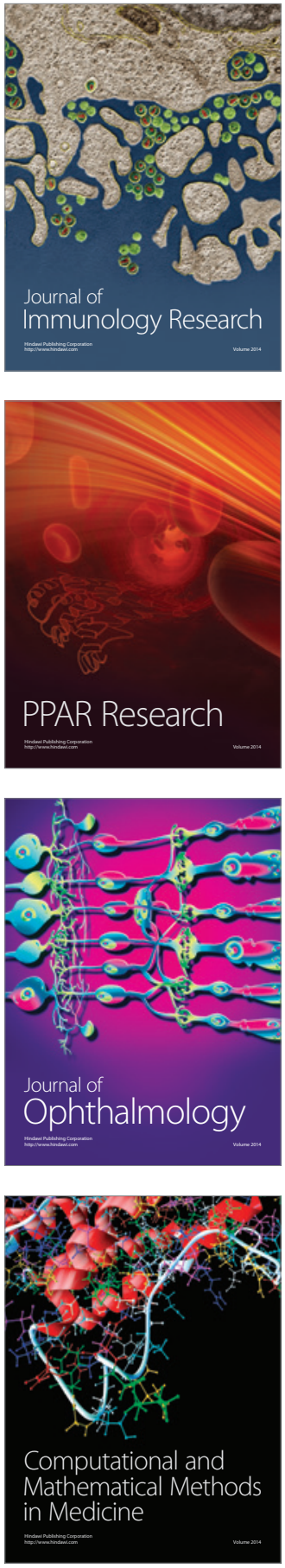

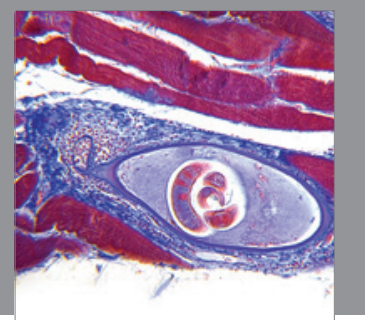

Gastroenterology

Research and Practice
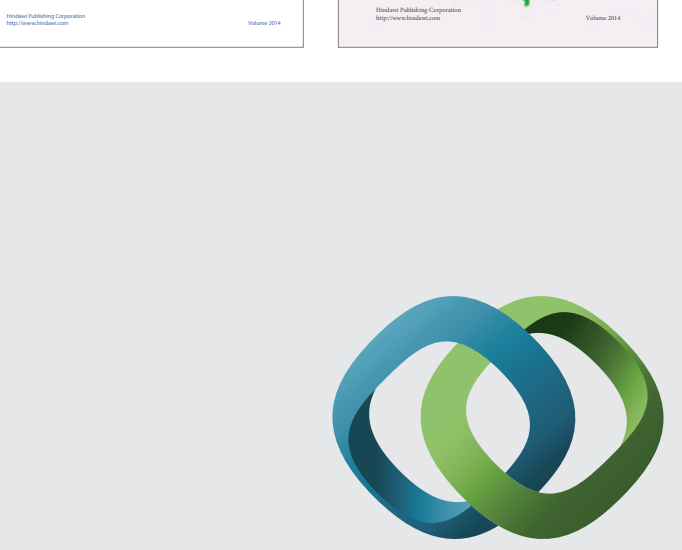

\section{Hindawi}

Submit your manuscripts at

http://www.hindawi.com
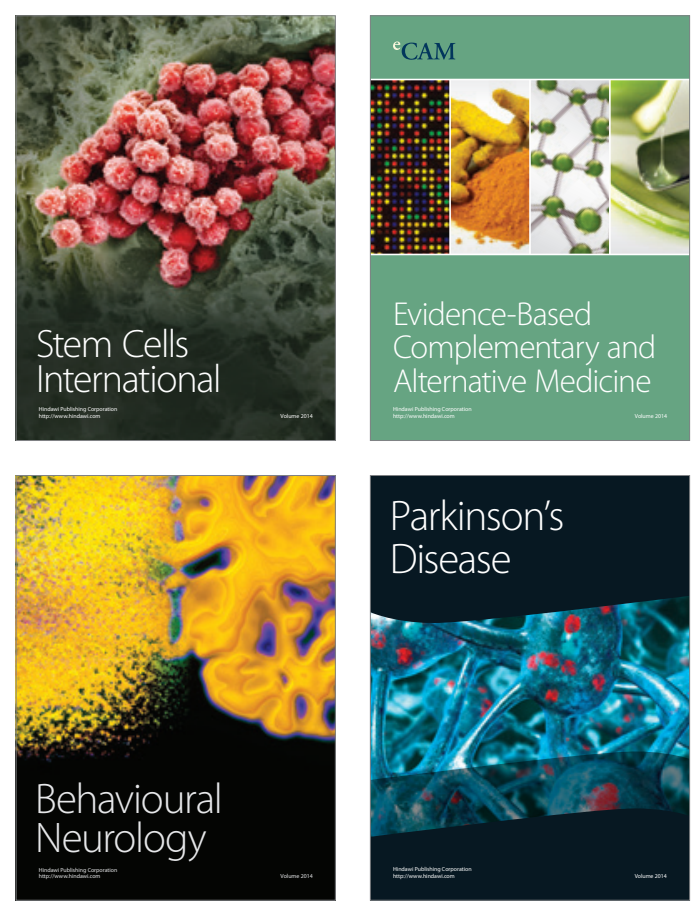

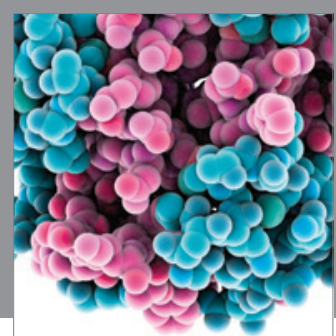

Journal of
Diabetes Research

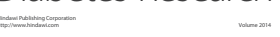

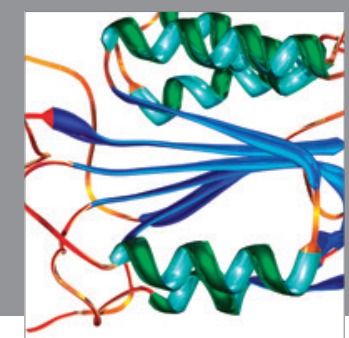

Disease Markers
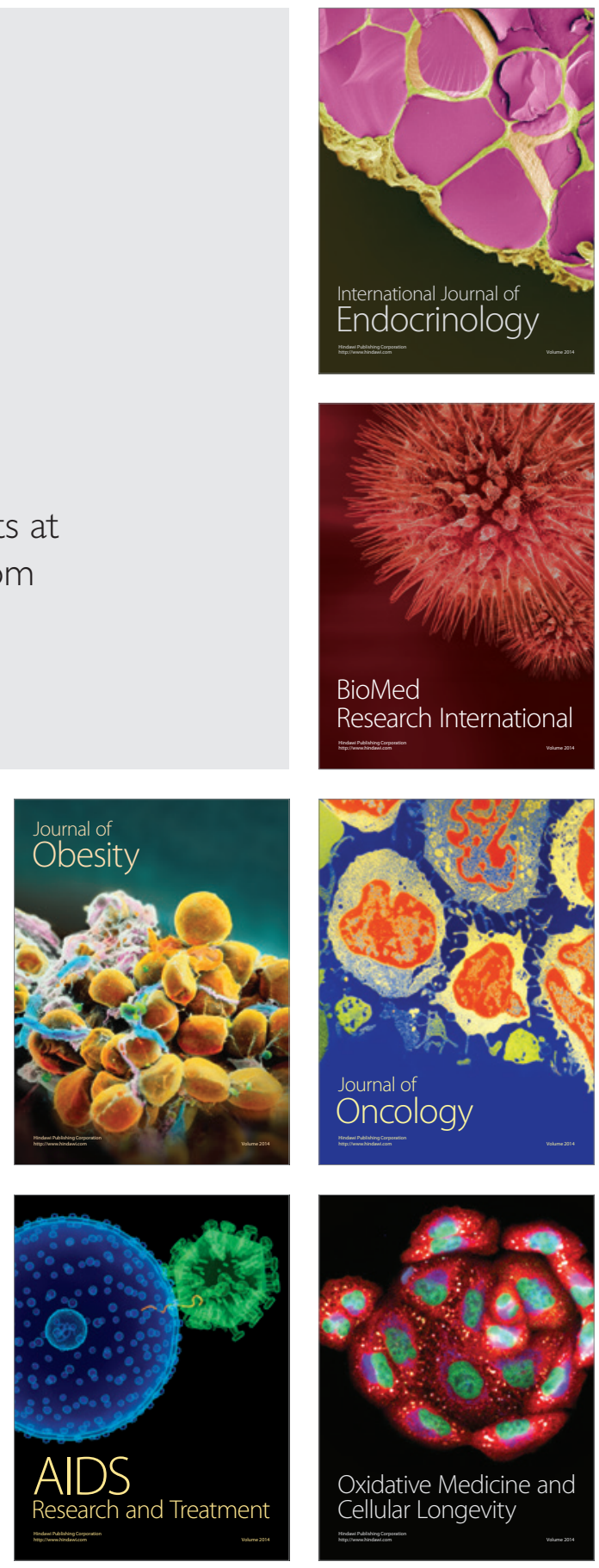